\title{
Some Remarks on Kappa-deformed Boost Transformations
}

\author{
Anatol Nowicki, Tomasz Masłowski \\ Institute of Physics, University of Zielona Góra \\ ul. Z. Szafrana 4a, 65-516 Zielona Góra, Poland \\ e-mail:A.Nowicki@if.uz.zgora.pl; T.Maslowski@proton.if.uz.zgora.pl \\ (Received: 23 March 2010; accepted: 8 July 2010; published online: 27 September 2010)
}

\begin{abstract}
We discuss the invariance of the action of the Lorentz algebra on the relativistic fourmomentum algebra under nonlinear transformations. In particular, we describe the global Lorentz boosts acting on four momenta in cases for classical and $\kappa$-deformed Poincaré relativistic symmetry.
\end{abstract}

Key words: symmetries, Hopf algebras, nonlinear transformations

\section{INTRODUCTION}

A description of modified fourdimesional $D=4$ classical relativistic symmetries using the concept of a Hopf algebra (quantum algebra) [1] was proposed in the early 1990 s using a model of so called $\kappa$-Poincaré algebra [2, 3]. It appears that one can modify an enveloping algebra of classical Poincaré Lie algebra introducing a new nonlinear basis. One can consider in some sense a minimal modification of Poincaré algebra considering nonlinear transformations of the four momenta generators leaving unchanged classical Lorentz algebra, i.e. $O(3)$-rotations and boost generators. This choice of basis is called the bicrossproduct basis [3].

In order to get a nontrivial Hopf algebra structure, one also has to deform the classical coalgebra structure introducing a nonsymmetric coproduct. In this description, noncommuting $D=4$ space-time is obtained as a dual Hopf algebra to $\kappa$-Poincaré algebra.

One of the most interesting physical problems is to find appropriate nonlinear transformations giving new momenta with physically reasonable properties. Further we shall briefly consider this problem.

\section{CLASSICAL AND $\kappa$-DEFORMED SYMMETRIES}

Introducing generators $\mathcal{M}_{i}$ describing $O(3)$-rotations and the boost generators $\mathcal{N}_{i}(i, j, k=1,2,3)$ one can write the Lie algebra commutation relations of the classical $D=4$ Poincaré algebra $\left(\mathcal{P}=\mathcal{L} \Subset T^{4}\right)$ in the form

- Lorentz algebra $\mathcal{L}$

$$
\begin{gathered}
{\left[\mathcal{M}_{i}, \mathcal{M}_{j}\right]=i \epsilon_{i j k} \mathcal{M}_{k}, \quad\left[\mathcal{M}_{i}, \mathcal{N}_{j}\right]=i \epsilon_{i j k} \mathcal{N}_{k},} \\
{\left[\mathcal{N}_{i}, \mathcal{N}_{j}\right]=-i \epsilon_{i j k} \mathcal{M}_{k} .}
\end{gathered}
$$

- Translation algebra $T^{4}$ generated by the commuting four momenta $\left[\mathcal{P}_{\mu}, \mathcal{P}_{v}\right]=0$ and

- covariance relations

$$
\begin{aligned}
& {\left[\mathcal{M}_{i}, \mathcal{P}_{j}\right]=i \epsilon_{i j k} \mathcal{P}_{k}, \quad\left[\mathcal{M}_{i}, \mathcal{P}_{0}\right]=0} \\
& {\left[\mathcal{N}_{i}, \mathcal{P}_{j}\right]=i \delta_{i j} \mathcal{P}_{0}, \quad\left[\mathcal{N}_{i}, \mathcal{P}_{0}\right]=i \mathcal{P}_{i}}
\end{aligned}
$$

The momentum variables fulfill the standard mass shell condition (Casimir quadratic in four momenta generators)

$$
C_{2}=\mathcal{P}_{0}^{2}-\overrightarrow{\mathcal{P}}^{2}=\mu^{2} c^{2}=i n v .
$$

where $c$ is the speed of light and $\mu$ - the rest mass.

There is a simple prescription for an extension of any Lie algebra to a Hopf algebra structure defining a trivial coalgebra structure [1] with a counit and an antipode. In particular, for the considered classical, nondeformed Poincaré algebra, $\mathcal{P}$, we define three algebra homeomorphisms $(\mu=0,1,2,3)$

$$
\begin{aligned}
- \text { coproduct } \Delta_{0}: \mathcal{P} \rightarrow \mathcal{P} \otimes \mathcal{P} & \\
\Delta_{0}(X) & =X \otimes 1+1 \otimes X, \quad \text { for } \quad X=\mathcal{M}_{i}, \mathcal{N}_{i}, \mathcal{P}_{\mu} .
\end{aligned}
$$


- antipode $S_{0}$ and counit $\epsilon$

$$
S_{0}(X)=-X, \epsilon(X)=0, \epsilon(1)=1,
$$

for $X=\mathcal{M}_{i}, \mathcal{N}_{i}, \mathcal{P}_{\mu}$.

It has appeared that this trivial extension of the Poincaré algebra to a Hopf algebra is not the only one. Introducing an additional deformation parameter $\kappa$ of the dimension of mass, one can slightly extend this Poincaré algebra Hopf structure in such a way that its coalgebraic sector becomes a nontrivial one.

The classical Poincaré algebra has a structure of a semidirect sum $\mathcal{P}=\mathcal{L} \Subset T^{4}$. It was shown that one can deform $\mathcal{P}$ in such a way that the semi-direct sum structure remains unchanged. It can be done using the so called bicrossproduct basis [3]. In this basis the Lorentz algebra commutation relations (1) do not change and the covariance relations (2) take the form

$$
\begin{aligned}
& {\left[\mathcal{M}_{i}, P_{j}\right]=i \epsilon_{i j k} P_{k},\left[\mathcal{M}_{i}, P_{0}\right]=0,\left[\mathcal{N}_{i}, P_{0}\right]=i P_{i} e^{-\frac{P_{0}}{2 \kappa c}},} \\
& {\left[\mathcal{N}_{i}, \mathcal{P}_{j}\right]=i e^{-\frac{P_{0}}{2 \kappa c}}\left[\delta_{i j}\left(\kappa c \sinh \left(\frac{P_{0}}{\kappa c}\right)+\frac{\vec{P}^{2}}{2 \kappa c}\right)-\frac{P_{i} P_{j}}{2 \kappa c}\right] .}
\end{aligned}
$$

In order to describe a nontrivial Hopf structure of this deformed $\kappa$-Poincaré algebra, we have to define coproduct $\Delta$, antipode $S$ and counit $\epsilon$ as follows

$$
\begin{gathered}
\Delta\left(\mathcal{M}_{i}\right)=\mathcal{M}_{i} \otimes 1+1 \otimes \mathcal{M}_{i}, \\
\Delta\left(P_{0}\right)=P_{0} \otimes 1+1 \otimes P_{0}, \\
\Delta\left(\mathcal{N}_{i}\right)=\mathcal{N}_{i} \otimes 1+e^{-\frac{P_{0}}{\kappa c}} \otimes \mathcal{N}_{i}+ \\
+\frac{1}{\kappa c} \epsilon_{i j k} e^{-\frac{P_{0}}{2 \kappa c}} P_{j} \otimes \mathcal{M}_{k}, \\
\Delta\left(\mathcal{P}_{i}\right)=\mathcal{P}_{i} \otimes e^{\frac{P_{0}}{2 \kappa c}}+e^{-\frac{P_{0}}{2 \kappa c}} \otimes P_{i},
\end{gathered}
$$

antipode and counit

$$
\begin{gathered}
S\left(\mathcal{M}_{i}\right)=-\mathcal{M}_{i}, \\
S\left(\mathcal{N}_{i}\right)=-e^{\frac{P_{0}}{\kappa c}} \mathcal{N}_{i}+\frac{1}{\kappa c} \epsilon_{i j k} e^{\frac{P_{0}}{2 \kappa c}} P_{j} \mathcal{M}_{k}, \\
S\left(P_{0}\right)=-P_{0}, \quad S\left(P_{i}\right)=-P_{i}, \\
\epsilon(X)=0, \quad X=\mathcal{M}_{i}, \mathcal{N}_{i}, P_{\mu} .
\end{gathered}
$$

The concept of coproduct allows us to consider the four momenta abelian algebra $T^{4}$ as a module of Lorentz algebra $\mathcal{L}$ so called $\mathcal{L}$-module.

\section{LORENTZ BOOST TRANSFORMATIONS}

Let us recall that the left action (right action) of Lorentz algebra on the momentum algebra $T^{4}$ is defined as follows [1]

$$
\begin{aligned}
& \stackrel{a d}{x \triangleright p}=x_{(1)} p S\left(x_{(2)}\right)=\sum_{k} x_{(1) k} p S\left(x_{(2) k}\right), \\
& a d \\
& p \triangleleft x=S\left(x_{(1)}\right) p x_{(2)}=\sum_{k} S\left(x_{(1) k}\right) p x_{(2) k},
\end{aligned}
$$

where $x \in \mathcal{L}, p \in T^{4}$ and (Sweedler's notation is used)

$$
\Delta(x)=\sum_{k} x_{(1) k} \otimes x_{(2) k} \equiv x_{(1)} \otimes x_{(2)} .
$$

It appears that in both cases of classical, nondeformed and $\kappa$-deformed Poincaré algebra in bicrossproduct basis we have

$$
\stackrel{a d}{x \triangleright p}=x_{(1)} p S\left(x_{(2)}\right)=[x, p] .
$$

Therefore, the left action (similarly right action) is classical, i.e. given by a commutator. From this relation follows a more general formula valid for an arbitrary function of four momenta $F\left(P_{0}, \vec{P}\right)$ namely

$$
e^{i \vec{\alpha} \overrightarrow{\mathcal{N}}} \stackrel{a d}{\triangleright} F\left(P_{0}, \vec{P}\right)=e^{i \vec{\alpha} \overrightarrow{\mathcal{N}}} F\left(P_{0}, \vec{P}\right) e^{-i \vec{\alpha} \overrightarrow{\mathcal{N}}},
$$

where $\vec{\alpha}=\alpha \vec{n}$ is the rapidity vector related to the velocity vector by a formula $\vec{v}=c \vec{n} \tanh \alpha$. In particular, for the nondeformed Poincaré algebra $\mathcal{P}$ (see Eqs. (1)-(2)) the relativistic boost transformations of the four momenta $\mathcal{P}_{\mu}$ are given by the formulae

$$
\mathcal{P}_{0}(\vec{\alpha})=\mathcal{P}_{0} \cosh \alpha-(\vec{n} \cdot \overrightarrow{\mathcal{P}}) \sinh \alpha,
$$

$$
\overrightarrow{\mathcal{P}}(\vec{\alpha})=\overrightarrow{\mathcal{P}}+\vec{n}\left((\vec{n} \cdot \overrightarrow{\mathcal{P}})(\cosh \alpha-1)-\overrightarrow{\mathcal{P}}_{0} \sinh \alpha\right) .
$$

From the form of relations (17)-(18) two main conclusions follow:

(i) for an arbitrary, nonlinear and invertible transformations of the four momenta basis $P_{\mu} \rightarrow \mathcal{P}_{\mu}$, these relations are invariant,

(ii) these relations become invariant for a large class of nonlinear transformations of the Lorentz generators $\overrightarrow{\mathcal{N}} \rightarrow \vec{L}$. 
Further we shall consider the case (i) and we assume that the invertible, nonlinear transformations are in the form (see Ref. [4])

$$
\begin{gathered}
\mathcal{P}_{0}=\kappa c B g \equiv \kappa c B\left(\frac{P_{0}}{\kappa c}, \frac{\vec{P}^{2}}{\kappa^{2} c^{2}}\right) g\left(\frac{P_{0}}{\kappa c}\right), \\
\overrightarrow{\mathcal{P}}=\vec{P} g\left(\frac{P_{0}}{\kappa c}\right),
\end{gathered}
$$

where invertible functions $g$ and $B$ depending on the parameter $\kappa$ satisfy the conditions:

$$
\lim _{\kappa \rightarrow \infty} g\left(\frac{P_{0}}{\kappa c}\right)=1 \text { and } \lim _{\kappa \rightarrow \infty} B\left(\frac{P_{0}}{\kappa c}, \frac{\vec{P}^{2}}{\kappa^{2} c^{2}}\right)=P_{0} .
$$

This form of transformations imply only changes in the covariance relations (6)-(7); therefore, the commutators $[\mathcal{N}, P]$ depend on functions $B$ and $g$ but the classical Lorentz algebra (1)-(2) is not changed.

The formulae (17)-(18) imply the following form of the boost transformations of nonlinearly transformed momentum $P_{\mu}$

$$
\begin{gathered}
B(\vec{\alpha}) W(\vec{\alpha})=B \cosh \alpha-\frac{(\vec{n} \vec{P})}{\kappa c} \sinh \alpha, \\
\vec{P}(\vec{\alpha})= \\
=W^{-1}(\vec{\alpha})(\vec{P}+\vec{n}[(\vec{n} \vec{P})(\cosh \alpha-1)-\kappa c B \sinh \alpha]),
\end{gathered}
$$

where:

$$
W(\vec{\alpha})=g(\vec{\alpha}) g^{-1} \text { and } X(\vec{\alpha}) \equiv e^{i \vec{\alpha} \overrightarrow{\mathcal{N}}} X e^{-i \vec{\alpha} \overrightarrow{\mathcal{N}}}, X=(g, B) .
$$

Using nonlinear transformations (21) the formula (3) becomes a deformed mass shell condition and it is given by the relation

$$
C_{2}=g^{2}\left(\kappa^{2} c^{2} B^{2}-\vec{P}^{2}\right)=C_{2}(\kappa)\left(1+\frac{C_{2}(\kappa)}{4 \kappa^{2} c^{2}}\right)=i n v .
$$

which holds for the special form of function $B$ satisfying additional requirements

$$
\begin{gathered}
B=f\left(\frac{P_{0}}{\kappa c}\right)+\frac{\vec{P}^{2}}{2 \kappa^{2} c^{2}} h\left(\frac{P_{0}}{\kappa c}\right), \\
g^{-2}+h^{-1}\left(2 f-h^{-1}\right)=0 .
\end{gathered}
$$

The $\kappa$-deformed Casimir is linear in the square of momentum and depends on the deformation parameter $\kappa$ and can be rewritten as follows

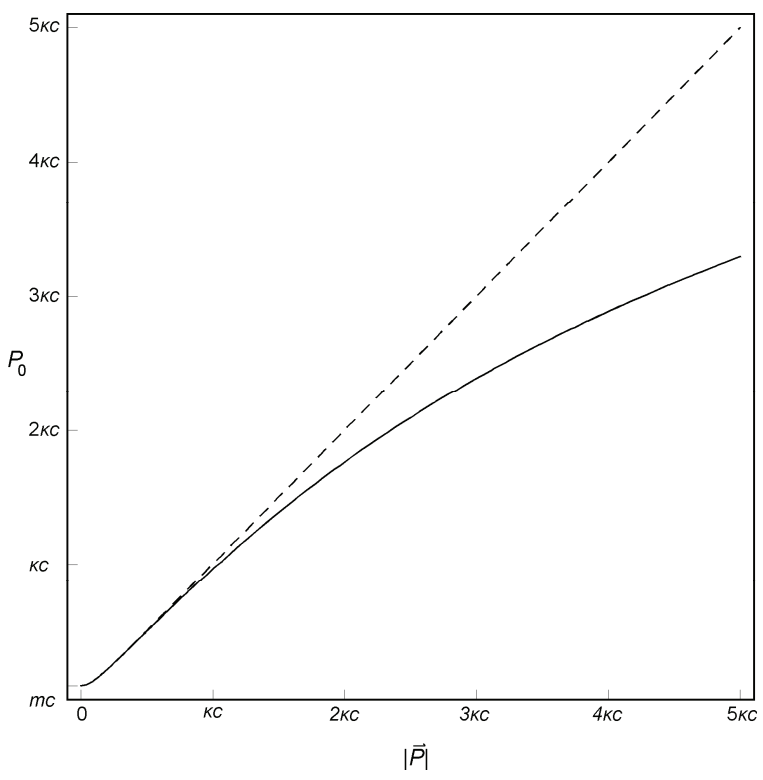

Fig. 1. The case (a) - dispersion relation

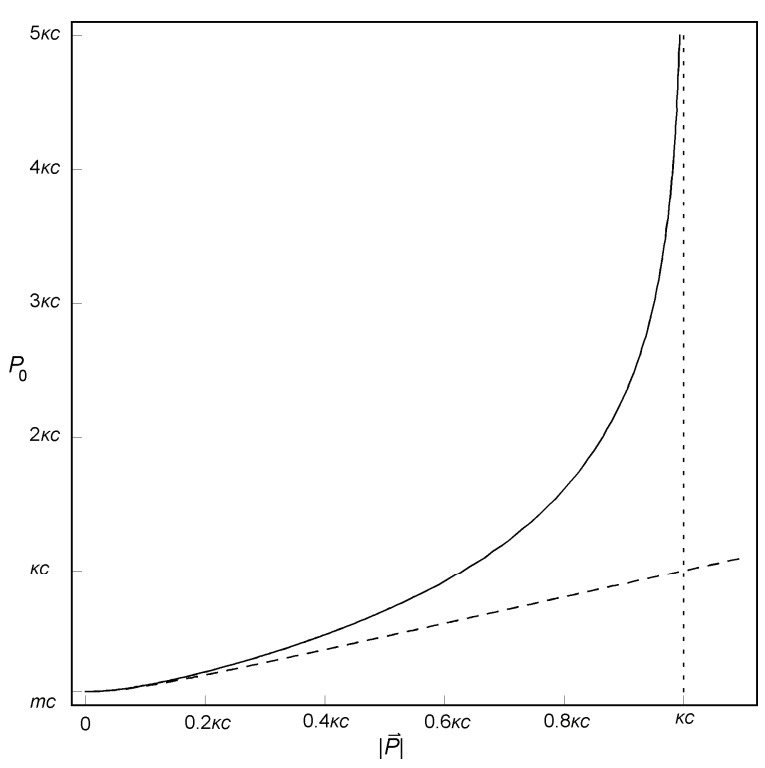

Fig. 2. The case (b) - dispersion relation

$C_{2}(\kappa)=2 \kappa^{2} c^{2}\left(g\left(h^{-1}-f\right)-1\right)-g h \vec{P}^{2}=M^{2} c^{2}=i n v$.

The form of the mass shell conditions and the physical properties of the momenta depend on the choice of the transformation functions $f, h, g$. In particular, the four momentum basis satisfying the relations (6)-(7) are given by the choice $(a): f=e^{x / 2} \sinh x, h=e^{x / 2}, \quad g=e^{x / 2}$, and the dispersion relation is plotted on Fig. (1). Similarly, most frequently used basis (bicrossproduct basis) [3] is given by (b): $f=1 / 2\left(1-e^{-2 x}\right), h=1, g=e^{x}$, see Fig. 2 (dashed lines on both plots represent classical behavior). 


\section{References}

[1] S. Majid, Foundations of Quantum Group Theory. Cambridge University Press, Cambridge (1995).

[2] J. Lukierski, A. Nowicki, H. Ruegg, Phys. Lett. B 293, 344 (1992).

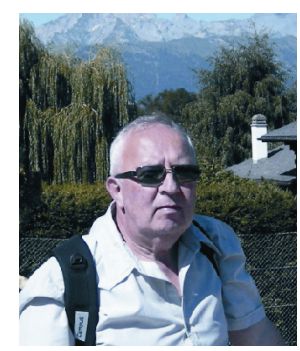

Dr. Hab. Anatol Nowicki works at the Institute of Physics of the University of Zielona Góra, Poland. He recived his Ph. D. (1977) and Dr. hab. (1988) degrees from the Wrocław University. His main areas of interest include the relativistic space-time symmetries, quantum groups and the application of the Hopf algebras methods to a description of non-commuting space-time.

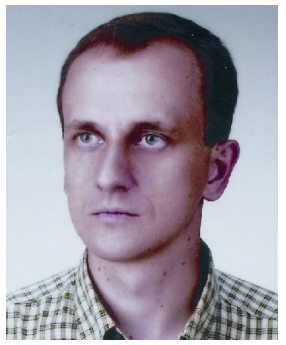

[3] S. Majid, H. Ruegg, Phys. Lett. B 334, 348 (1994).

[4] J. Lukierski, A. Nowicki, Int. J. Mod. Phys. A 18, 7 (2003). ters methods to a desciption of non-commuting space-tine.

Dr. TOMASz MasŁowsKI works at the Institute of Physics of the University of Zielona Góra, Poland. He was graduated from the University of Warsaw with a Ph.D. (2006) in theoretical physics. His field of interest are QCD, renormalization group methods and presently quantum field theories with $\kappa$-deformed Poincaré symmetry. 